\title{
Managing mental health challenges faced by healthcare workers during covid-19 pandemic
}

\author{
Neil Greenberg and colleagues set out measures that healthcare managers need to put in place \\ to protect the mental health of healthcare staff having to make morally challenging decisions
}

\section{Neil Greenberg professor of defence mental health ${ }^{1}$, Mary Docherty consultant liaison psychiatrist ${ }^{2}$, Sam Gnanapragasam NIHR academic clinical fellow in psychiatry ${ }^{2}$, Simon Wessely regius professor of psychiatry $^{1}$}

${ }^{1}$ NIHR Health Protection Research Unit in Emergency Preparedness and Response, King's College London, London, UK; ${ }^{2}$ South London and the Maudsley NHS Trust, London, UK

\begin{abstract}
The covid-19 pandemic is likely to put healthcare professionals across the world in an unprecedented situation, having to make impossible decisions and work under extreme pressures. These decisions may include how to allocate scant resources to equally needy patients, how to balance their own physical and mental healthcare needs with those of patients, how to align their desire and duty to patients with those to family and friends, and how to provide care for all severely unwell patients with constrained or inadequate resources. This may cause some to experience moral injury or mental health problems.
\end{abstract}

\section{Moral injury}

Moral injury, a term that originated in the military, can be defined as the psychological distress that results from actions, or the lack of them, which violate someone's moral or ethical code. ${ }^{1}$ Unlike formal mental health conditions such as depression or post-traumatic stress disorder, moral injury is not a mental illness. But those who develop moral injuries are likely to experience negative thoughts about themselves or others (for example, "I am a terrible person" or "My bosses don't care about people's lives") as well as intense feelings of shame, guilt, or disgust. These symptoms can contribute to the development of mental health difficulties, including depression, post-traumatic stress disorder, and even suicidal ideation. ${ }^{2}$ Equally, some people who have to contend with significant challenges, moral or traumatic, experience a degree of post-traumatic growth, ${ }^{3}$ a term used to describe a bolstering of psychological resilience, esteem, outlook, and values after exposure to highly challenging situations. Whether someone develops a psychological injury or experiences psychological growth is likely to be influenced by the way that they are supported before, during, and after a challenging incident.

Moral injury has already been described in medical students, who report great difficulty coping with working in prehospital and emergency care, ${ }^{4}$ where they were exposed to trauma that they felt unprepared for. This may be similar to the unprecedented nature of the challenges healthcare staff are currently facing. In the UK, most NHS staff may have felt, with some justification, that with all its faults, the NHS gives the sickest people the greatest chance of recovery. As such, staff should and usually do feel that it is something to be proud of.

The huge current effort to ensure adequate staffing and resources may be successful, but it looks likely that during the covid-19 outbreak many healthcare workers will encounter situations where they cannot say to a grieving relative, "We did all we could" but only, "We did our best with the staff and resources available, but it wasn't enough." That is the seed of a moral injury. Not all staff members will be adversely affected by the challenges ahead (table 1) but no one is invulnerable, and some healthcare workers will hurt, perhaps for a long time, unless we begin now to prepare and support our staff.

\section{Early support}

Several potential mechanisms can help mitigate the negative moral effects of the current situation. All healthcare workers need to be prepared for the moral dilemmas they are going to face during the covid-19 pandemic. We know that properly preparing staff for the job and the associated challenges reduces the risk of mental health problems. ${ }^{6}$ They should not be given false reassurance but a full and frank assessment of what they will face, delivered without euphemisms and in plain English. To do anything else may add to the feelings of anger when reality bites.

As the situation progresses, team leaders should help staff make sense of the morally challenging decisions being made. This could be achieved by using discussions based on Schwarz rounds, ${ }^{7}$ which provide a forum for healthcare staff from all 
backgrounds to safely discuss the emotional and social challenges of caring for patients. The discussion should be led by team leaders and could be done remotely if needed.

Avoidance is a core symptom of trauma, so team leaders should reach out to staff who are just "too busy" or repeatedly "not available" to attend these discussions. Most people find that support from their colleagues and immediate line manager protects their mental health. ${ }^{8}$ Staff members who persistently avoid meetings or become overly distressed may require and welcome sensitive discussion and support from a suitably experienced person such as their team leader, trained peer supporter, or chaplain. If their distress is severe or persistent they should be actively supported or, for more serious cases, referred for professional mental health support. Single session psychological debriefing approaches should not be used as they may cause additional harm. ${ }^{9}$

Routine support processes (such as peer support programmes) available to healthcare staff should include a briefing on moral injuries, as well as an awareness of other causes of mental ill health and what to look out for. Even the most resilient team members may become overwhelmed by situations that have personal relevance, such as providing care for someone who reminds them of a relative or a friend. Even staff members experienced in breaking bad news to relatives may be overcome by having to do this many times a day for weeks on end, especially if they have genuine feelings of guilt. In such situations both moral injury and burnout may affect mental health.

Although there is a wealth of evidence that having a supportive supervisor protects your mental health, ${ }^{10}$ supervisors are human too. As such, more senior managers should keep an active eye on more junior ones and check how they are doing. If they show signs of presenteeism - that is, working less effectively because of poor mental health-this will directly affect the operational capability and health of all team members, and thus early identification and support are key.

\section{After care}

Once the crisis is over, supervisors should ensure that time is made to reflect on and learn from the extraordinarily difficult experiences to create a meaningful rather than traumatic narrative. The National Institute for Health and Care Excellence recommends "active monitoring" of staff to ensure that the minority who become unwell are identified and assisted to access evidence based care. ${ }^{11}$ Clinicians who provide care for moral injuries and associated mental illness should also be aware of the potential to avoid speaking about guilt and shame and focus on other stressors during therapy. This therapeutic avoidance can lead to poorer outcomes. ${ }^{12}$

These are extraordinary times. There is a pressing need to ensure that the tasks ahead do not cause long lasting damage to healthcare staff. They will be the heroes of the day, but we will need them for tomorrow. For hundreds of years, the military have recognised the critical role of junior leaders in maintaining the will and capability of troops to continue to fight even in the most arduous of conditions. Similarly, healthcare managers in supervisory positions must now acknowledge the challenge staff face and minimise the psychological risk inherent in dealing with difficult dilemmas, and those in charge of resources must provide them with the opportunity to do so.

\section{Key messages}

Healthcare staff are at increased risk of moral injury and mental health problems when dealing with challenges of the covid-19 pandemic Healthcare managers need to proactively take steps to protect the mental wellbeing of staff

Managers must be frank about the situations staff are likely to face Staff can be supported by reinforcing teams and providing regular contact to discuss decisions and check on wellbeing

Once the crisis begins to recede, staff must be actively monitored, supported, and, where necessary, provided with evidence based treatments

Contributors and sources: This article is based on our collective professional experience and a review of published material. The authors have extensive experience of conducting research during and after major incidents, including the London bombings, Ebola, and infections such as swine flu. NG is a specialist in the understanding and management of psychological trauma, occupational mental ill health, and post-traumatic stress disorder and was president of the UK Psychological Trauma Society during 2014-17. MD is an expert clinical adviser to NHS England's adult mental health clinical policy and strategy group. SG has carried out research in disaster risk reduction with Public Health England colleagues and is currently undertaking clinical work in liaison psychiatry at King's College Hospital. SW is past president of the Royal College of Psychiatrists and president of the Royal Society of Medicine. He serves on numerous government committees for the Department of Health, Cabinet Office, and Ministry of Defence and recently chaired the independent review of the Mental Health Act. The article was conceptualised by SW and NG. The original draft was led by NG. The manuscript was reviewed and edited by NG, SW, MD, and SG prior to submission. NG is the guarantor.

Competing interests: We have read and understood BMJ policy on declaration of interests and have the following interests to declare: NG runs a psychological health consultancy that provides resilience training for a wide range of organisations, including a few NHS teams. The work was supported by the National Institute for Health Research (NIHR) Health Protection Research Unit in Emergency

Preparedness and Response at King's College London, in partnership with Public Health England and in collaboration with the University of East Anglia and Newcastle University. The views expressed are those of the authors and not necessarily those of the NHS, NIHR, Department of Health and Social Care, or Public Health England.

Provenance and peer review: Not commissioned; externally peer reviewed.

Litz BT, Stein N, Delaney E, etal . Moral injury and moral repair in war veterans: a preliminary model and intervention strategy. Clin Psychol Rev 2009;29:695-706. 10.1016/j.cpr.2009.07.003 19683376

2 Williamson V, Stevelink SAM, Greenberg N. Occupational moral injury and mental health: systematic review and meta-analysis. Br J Psychiatry 2018;212:339-46. 10.1192/bjp.2018.55 29786495

3 Brooks S, Amlôt R, Rubin GJ, Greenberg N. Psychological resilience and post-traumatic growth in disaster-exposed organisations: overview of the literature. BMJ Mil Health 2020;166:52-6. 10.1136/jramc-2017-000876 29420257

4 Murray E, Krahé C, Goodsman D. Are medical students in prehospital care at risk of moral injury? Emerg Med $J$ 2018;35:590-4

10.1136/emermed-2017-20721610.1136/emermed-2017-207216 29945983

5 Syracuse University. Moral injury project. http://moralinjuryproject.syr.edu/about-moralinjury/

6 Iversen AC, Fear NT, Ehlers A, etal . Risk factors for post-traumatic stress disorder among UK armed forces personnel. Psychol Med 2008;38:511-22. $10.1017 /$ S0033291708002778 18226287

7 Flanagan E, Chadwick R, Goodrich J, Ford C, Wickens R. Reflection for all healthcare staff: a national evaluation of Schwartz rounds. J Interprof Care 2020;34:140-2. 10.1080/13561820.2019.1636008 31390225

8 Greenberg N, Thomas S, Iversen A, Unwin C, Hull L, Wessely S. Who do military peacekeepers want to talk about their experiences? Perceived psychological support of UK military peacekeepers on return from deployment. $J$ Ment Health 2003;12:565-73. 10.1080/09638230310001627928

9 Brooks SK, Rubin GJ, Greenberg N. Traumatic stress within disaster-exposed occupations: overview of the literature and suggestions for the management of traumatic stress in the workplace. Br Med Bull 2019;129:25-34. 10.1093/bmb/ldy040 30544131

10 Jones N, Seddon R, Fear NT, McAllister P, Wessely S, Greenberg N. Leadership, cohesion, morale, and the mental health of UK armed forces in Afghanistan. Psychiatry 2012;75:49-59. 10.1521/psyc.2012.75.1.49 22397541

11 National Institute for Health and Care Excellence. Post-traumatic stress disorder. NG116. 2018. https://www.nice.org.uk/guidance/ng116

12 Salkovskis PM, Clark DM, Hackmann A, Wells A, Gelder MG. An experimental investigation of the role of safety-seeking behaviours in the maintenance of panic disorder with 
agoraphobia. Behav Res Ther 1999;37:559-74 10.1016/S0005-7967(98)00153-3 10372469
Published by the BMJ Publishing Group Limited. For permission to use (where not already granted under a licence) please go to http://group.bmj.com/group/rights-licensing/ permissions 


\section{Table}

\section{Table 1| Potential for moral injury: analogous examples of events or actions in military settings ${ }^{5}$ and the covid-19 pandemic}

\section{Military examples}

Expected healthcare examples

Following orders that were illegal, immoral, or against the Rules of Engagement Following clinical decisions by others that the individual believes were unethical, or Geneva Convention

Failing to report knowledge of a sexual assault or rape committed against yourself, a fellow service member, or civilians

Change in belief about the necessity or justification for a conflict, during or after military service

Putting a colleague in serious danger because of own inexperience or indecision

Returning home from deployment and hearing of the atrocities that occurred "on your watch" immoral, or against guidance from registered professional bodies

Failing to report serious clinical incidents, near misses, or bullying of yourself, colleagues, or patients

Change in belief about the necessity or justification for treatment plans or protocols that have affected people's lives

Putting patients or colleagues in danger because of your inexperience, indecision, or working outside your normal competency

Being told that you are unable to treat a seriously ill civilian (especially someone Having to choose which of two equally sick patients is provided with specific care, you perceive as vulnerable, such as a child) brought to the gates of your camp, one of whom does not survive, because of the non-availability of healthcare who subsequently dies equipment

Giving orders during combat that result in the injury or death of a fellow service Giving clinical orders or establishing protocols that result in the death of colleagues member or innocent civilians or patients

Using deadly force in combat and causing the harm or death of civilians, knowingly but without alternatives, or unintentionally

Responding acutely in medical emergency and causing the harm or death of patients, knowingly but without alternatives, or unintentionally

Feeling let down when the chain of command does not provide you with adequate reinforcements 